\title{
The Nature and Origin of Inertia
}

\author{
Nader Butto \\ Petah-Tikva, Israel \\ Email: nader.butto@gmail.com
}

How to cite this paper: Butto, N. (2021) The Nature and Origin of Inertia. Journal of High Energy Physics, Gravitation and Cosmology, 7, 761-772. https://doi.org/10.4236/jhepgc.2021.72044

Received: March 2, 2021

Accepted: April 27, 2021

Published: April 30, 2021

Copyright (c) 2021 by author(s) and Scientific Research Publishing Inc. This work is licensed under the Creative Commons Attribution International License (CC BY 4.0).

http://creativecommons.org/licenses/by/4.0/

\begin{abstract}
This paper aims to present a new theory that explains the mechanism of inertia at providing a satisfying explanation for the yet unknown mechanism for inertia. By considering the vacuum as a liquid with a measurable density, hydrodynamics laws are used to describe the behaviour of the vacuum when it is dragged by moving body. The inertia is the result of the initial resistance between the moving bodies against the static vacuum. The moving body drags the resisting vacuum during acceleration, till the point that the vacuum travels with the moving body and has the same velocity. When the body decelerates, the vacuum continues to flow and to push the body at the same direction of the original flow till its complete stop. Formulations based on Planck theory derived to prove its equivalence to Newton inertia law. Formulation based on hydrodynamics is derived to confirm the theory that the force exerted by the vacuum on static body in gravity and on moving body in inertia is equivalent to Newton law. The strong equivalence principle is reaffirmed and, consequently, Einstein's equations are preserved.
\end{abstract}

\section{Keywords}

Inertia Force, Vacuum Density, Drag Force, Newton Inertia Law, Gravitation Force

\section{Introduction}

Inertia is one of the main manifestations of mass, which is a quantitative property of physical systems. It is the resistance force of any physical object to any change in its state of movement. This includes changes in the speed, direction, or resting state of the object. Inertia is also defined as the tendency of objects to keep moving in a straight line at a constant speed. There is no measurable difference between the gravitational mass and the inertial mass.

Several theories have been put forward in attempts to explain inertia. However, most of the mainstream physics is based on Newtonian and Einstein's physics 
which accepts that gravity and inertia are identical, however, the machinery of gravitation and inertia are completely unknown.

In fact, inertia is considered to be a fundamental property that has not been properly addressed by Newton's first law and law of gravity, and till now the fundamental problem about the equivalence of Gravity and Inertia still unresolved. The equivalence principle asserts that gravitational mass and inertial mass are identical. The gravitational force we experience on Earth is identical to the force we would experience in a spaceship accelerating at $1 \mathrm{~g}$.

Newton's Laws of Motion are still used to describe the motion of objects and how they are affected by the applied forces on them. Isaac Newton defined inertia as his first law in his Philosophiz Naturalis Principia Mathematica, which states: [1]

"The innate force of matter, is a power of resisting by which everybody, as much as in it lies, endeavours to preserve its present state, whether it be of rest or of moving uniformly forward in a straight line".

Einstein's concept of inertia according to Special Relativity as proposed in his 1905 paper entitled "On the Electrodynamics of Moving Bodies" remained unchanged from Newton's original meaning.

The principle of relativity could only apply to reference frames that were inertial in nature (meaning when no acceleration was present). In order to overcome this limitation, he proceeded with his general relativity ("The Foundation of the General Theory of Relativity," 1916), introducing the new concept of "curvature" of space-time, instead of the more traditional system of forces understood by Newton [2].

However, in general relativity, there is no mechanism that generates a force to oppose deviations of test particles from geodesic motion.

Einstein redefined the concept of "inertia" in terms of geodesic deviation that describes the tendency of objects to approach or recede from one another while moving under the influence of a spatially varying gravitational field. Gravitational radiation is generated in situations where the curvature of space-time is oscillating, such as is the case with co-orbiting objects.

The result of this is that inertia is the gravitational coupling between matter and space-time, the energy density of the zero-point field would generate an enormous space-time curvature, akin to a huge cosmological constant. This is, of course, true in the standard interpretation of mass-energy.

Inertia has not been properly addressed by quantum field theory or superstring theory either.

Quantum physicists have so far been unable to use quantum theory as a lever to tease apart the behaviour of inertial and gravitational mass. They point out the important distinction between kinematics, which is concerned purely with motion not how it arises, and dynamics which focuses on the origin of motion. In the classical world, this has no bearing on the effects of inertial and gravitational mass.

From the other hand According to Higgs theory any field that interacts with 
the Higgs field acquires a mass [3].

Therefore, Higgs field gives masses to the elementary particles. The acquisition of mass-energy via a Higgs field may still allow for a mechanism to generate an inertial reaction force upon acceleration. However, there is no mechanism that explains how the Higgs-induced mass gives the property of inertia.

According to Stochastic Electrodynamics (SED) theory, [4] inertia may originate from interactions between the electromagnetic zero-point field of the quantum vacuum and the quarks and electrons constituting matter. In Stochastic Electrodynamics (SED), which is an extension of the de Broglie-Bohm interpretation of quantum mechanics, [5] [6] describes energy contained in the electromagnetic vacuum at absolute zero as a stochastic, fluctuating zero-point field. Modern approaches to SED consider the quantum properties of waves and particles as well-coordinated emergent effects resulting from deeper (sub-quantum) nonlinear matter-field interactions [7] [8] [9] [10].

However, SED-based calculations and SED-related claims related to inertia and gravity are still controversial and some have been subject to published criticism [4] [11].

Another theoretical breakthrough by Puthoff is the derivation of Newton's Law $(F=m a)$ from ZPE electrodynamics. It appears to be related to the known distortion of the zero-point spectrum in an accelerated reference frame. Thus, the resistance to acceleration defines the inertia of matter and it appears to be an electromagnetic resistance. Therefore, the inertia effect is a distortion at high frequencies whereas, the gravity effect has been shown to be low frequency effect, according to the Puthoff theory.

Physicists and mathematicians appear to be less inclined to use the popular concept of inertia as "a tendency to maintain momentum" and instead favour the mathematically useful definition of inertia as the measure of a body's resistance to changes in velocity or simply a body's inertial mass.

However, since classical free fall motions (for the quantum case), although accelerating ones, do not depend on the test mass $\left(t_{f}=(2 h / g)^{1 / 2}\right.$, one may think of relating them to fundamental predynamical (geometrical) properties of the universe.

As no alternate mechanism has been readily accepted, and it is now generally accepted that there may not be one which we can know, the term "inertia" has come to mean simply the phenomenon itself, rather than any inherent mechanism. Thus, ultimately, "inertia" in modern classical physics has come to be a name for the same phenomenon described by Newton's First Law of Motion, and the two concepts are now considered to be equivalent.

This paper aims to provide a satisfying theory to explain the mechanism of inertia based on the basic assumption that the universe is immersed in a vacuum with a well-defined density that behaves as a fluid and that the accelerated object moving through the fluid vacuum will experience a bath of radiation resulting from the quantum vacuum and the zero-point field will yield a non-zero Poynting vector. 
Scattering of this radiation by the quarks and electrons constituting matter would result in an acceleration-dependent reaction force that would appear to be the origin of inertia of matter [12] [13].

Furthermore, the vacuum is dragged by the body, and travel with the moving body, when the body is stopped the vacuum continue to move pushing the body and slows its flow until its total stop.

The physical and mathematical basis for the origin of inertia is described. Applying both quantum mechanics and hydrodynamics laws the inertia of matter can be successfully translated into the more simple and precise formulation.

\section{The Nature of Quantum Vacuum}

It seems that there is clear boundary between classical behavior of macroscopic bulk matter objects governed by classical physics and the behavior of microscale objects governed by quantum physics. Assembling a sufficiently large number of quantum objects together seems invariably to produce a classically behaved object, thus in order to understand the classical behaviour we must consider the quantum interaction of elements with the vacuum.

By doing so, the borderline between the two different regimes from quantum to classical becomes precisely identifiable.

Understanding the nature of the vacuum and its interaction with matter allows us to achieve a clearer understanding of the nature of gravitation and inertia.

A vacuum by definition has no mass, since there is nothing in it to produce mass. The vacuum extends everywhere, has no size, shape, center, direction, time, or extent, and is immovable.

According to Maxwell's equations the speed of light is determined exclusively by the permeability and permittivity of space, therefore, space is established to be as the "light medium" and light is a disturbance in space.

The theory of relativity rejects the idea of "light medium" and, therefore, it also rejects the fundamental sense of inertia.

During the early years of quantum mechanics, Paul Dirac theorized that the vacuum was actually filled with particles in negative energy states [14], thus giving rise to the concept of the "physical vacuum" which is not empty at all.

Physical theories predict that on an infinitesimally small scale, far smaller than the diameter of atomic nucleus, quantum fluctuations produce a foam of erupting and collapsing, virtual particles, visualized as a topographic distortion of the fabric of space time.

Quantum theory requires empty space to be filled by pairs of created and annihilated virtual particles. It is predicted that these invisible particles could materialize for a short time and exert a measurable force. Therefore, the physical vacuum is assumed to be a non-trivial medium, not empty but rather filled with quantum mechanical zero-point energy and characterized as behaving like a frictionless fluid with extremely low viscosity, in which one can associate a certain 
energy and density with extremely high thermal conductivity.

Therefore, the vacuum energy has real physically observable consequences, and its properties can be observed as having real physical effects [15] [16].

Given the relative familiarity and accessibility of fluid systems, it is only natural to draw analogies with them as one attempts to come to grips with the oddities of quantum mechanics.

The flow of such vacuum energy has far greater penetrating power than the gamma radiation, and can even pass through the earth and planets.

Therefore, the vacuum made off massless photons which are zero-rest mass particles, with minimum electromagnetic wavelength limit $=\lambda_{p}=4.051 \times 10^{-35}$ $\mathrm{m}$.

The highest frequency possible, that is, the shortest wavelength limit is equal to the dimension of the unit element making up space-time itself, equal to Planck length, therefore, maximum universal cosmic radiation frequency limit called Planck frequency $=c / \lambda_{p}=7.4 \times 10^{42} \mathrm{~Hz}$. This is the frequency at which unification of all other forces occurs, which its value in electron volts is would be $E / e=4.44665 \times 10^{17} \mathrm{Gev}$ equivalent to $5.18 \times 10^{30}$ Kelvin.

The relationship between wavelength and energy of, $E$, is described by the equation, $E=h c / \lambda$, where $h$ is Planck's constant ( $h=6.625 \times 10^{-34}$ Joule-seconds or $\mathrm{J} \mathrm{s}$ ) and $c$ is the speed of light. By replacing the constants $h$ and $c$ with their respective values, we see that $E=1.986 \times 10^{-25}$ Joule-meters or $\mathrm{J} \mathrm{m} / \lambda$.

This is the upper energy levels and frequency limits in the universe that can interact with matter. Shorter wavelengths than Planck length have no effect on matter, nor can they be generated by any interaction with matter, in other words, they cannot manifest in our physical reality.

Such radiation made of photons, is found everywhere in space and its maximum frequency exceeds by far the maximum frequency which we are able to detect with our present detectors. The energy of each individual photon is a crucial component of the momentum necessary to create pressure for gravity and inertia to be possible.

Such photons have the properties of energy and momentum, thus exhibit the property of mass as they travel against moving mass, upon colliding with a mass, deliver a portion of their momentum to that mass and push it backward with a force proportional to the extension or the volume and density (the mass) of the considered molecule.

The fact that electromagnetic radiation exerts a pressure upon any surface exposed to it was deduced theoretically by the father of electromagnetic theory James Clerk Maxwell in 1871, and later on, proven experimentally by Lebedev in 1900 and by Nichols and Hull in 1901.

\section{The Essence of Inertia}

When the velocity of an object changes it is said to be accelerating. Acceleration is the rate of change of velocity with time. When the changes an increasing speed 
is called acceleration, while decreasing speed called deceleration, changing direction called centripetal acceleration.

Newton originally viewed the phenomenon of inertia he described in his First Law of Motion as being caused by "innate forces" inherent in matter, which resisted any acceleration.

There are unusual effects that occur in accelerated frames, uniformly accelerating detector in the vacuum will experience inertia and temperature known as Unruh effect.

The Unruh effect is the prediction that an accelerating observer will observe a thermal bath, like blackbody radiation, whereas an inertial observer would observe none [17].

In other words, the background appears to be warm from an accelerating reference frame. This would seem to be more of an intrinsic origin of inertia related to the mass itself.

The inertia can be better explained relying on the effect of the quantum vacuum energy on matter through the effect of ultra-high frequency radiation on matter.

The radiation pressure exerted by such ultra-high frequency radiation, would cause gravity and inertia effect, since such radiation would penetrate any matter and act all over its constituent particles, not just its surface.

The interaction between the flowing virtual photons against inertial mass creates the gravitation force, while the interaction of a moving mass against the quantum vacuum which is made of virtual photons creates inertia or acceleration. They have the same magnitude with one difference, the flow that creates the gravity force is always at speed of light, while the inertia related to speed of body in space. In both cases radiation pressure is present due to longitudinal waves with Pointing vector which oscillations in the direction of flow in the case of gravity and in the opposite direction of the body movement in the case of inertia. The Poynting vector describes the flow of energy through a surface in terms of electric and magnetic properties and has the dimensions of power per unit area. Poynting vector can travel through vacuum, and its magnitude is always positive creating radiation pressure. Radiation pressure in Pascals $\left(\mathrm{N} / \mathrm{m}^{2}\right)$ is equal to the time averaged Poynting vector magnitude divided by the speed of light when gravitation is considered, and average velocity of moving body in case of inertia.

\section{Newton Law of Inertia Derived from Quantum Mechanics}

According to Newton, an object will maintain its velocity unless acted on by a net external force, whether it results from gravity, friction, contact, or some other force.

The newton force of gravitation takes place on the quantum level. In order to prove this, we should derive newton law from quantum mechanics.

It is well accepted that all matter, boils down to different structures made up 
of the same constituent particle (whatever it might be). Such building block of matter, must have the same energy and size for all existing matter.

The interaction between waves and accelerating body, which is made of particles, takes place in a particular frequency or close harmonics, that their wavelength equal to the particles' diameter, so that the waves would be absorbed by such building block particles which happen to be in the way of the travelling body. Applying Planck units for a single travelling electromagnetic wave, travelling at the speed of light $c$, we have:

$$
\lambda_{p} \equiv\left(G h / c^{3}\right)^{1 / 2}=4.05096 \times 10^{-33} \mathrm{~cm}
$$

Minimum electromagnetic wavelength limit $=\lambda_{p}=4.051^{-35} \mathrm{~m}$.

The minimum building block particle diameter $=4.051^{-35} \mathrm{~m}$, which is in the range of Planck wavelength.

Since the number of such particles is directly proportional to the number of atoms constituting the whole body, and hence proportional to its total atomic mass, the net effect of radiation pressure over the body would be exactly equal to inertia.

The interaction between the moving object and the vacuum takes place in units of Planck's constant times the frequency. Therefore, the energy transferred to the matter is

$$
E=h f
$$

and it should give the same value of inertia according to Newton law $F=$ ma. If

$$
E=m c^{2}=h f=F \lambda
$$

where $c=$ velocity of light, $f=1 / t=$ frequency, $F=$ force, $\lambda=$ wavelength (distance) thus, the force would be:

$$
F=E / \lambda=m c^{2} / \lambda
$$

If $c=\lambda / t$ then Replace the value of $c^{2}$ in formula

$$
F=m \lambda^{2} / t^{2} \lambda=m \lambda / t^{2}
$$

If $\lambda=c t$ then

$$
F=m \lambda / t^{2}=m c t / t^{2}=m c / t
$$

Since $c t=a$, acceleration. Therefore

$$
F=m a
$$

\section{Newton Law Derivation from Hydrodynamics Laws}

Any motion relative to quantum vacuum requires force and it is accompanied by acceleration.

Considering the quantum vacuum as a fluid the moving body will interact with the relative static quantum vacuum, exerts a real effect of a positive force due to the momentum given up during the interaction with matter.

When momentum is exchanged with matter the electromagnetic wave be- 
haves as a particle, that takes place on the range of frequency wavelength equal to the fundamental building block size of matter. Therefore, the ultra-high frequency of the quantum vacuum energy will penetrate the body until the level of elementary particles which are made as spheres and exert an opposing force. Considering the vacuum as a fluid, hydrodynamic laws are applied to explain gravity and inertia.

In hydrodynamics, if a sphere moving in liquid medium with density $\rho$ and area $A$ and the velocity of the sphere relative to the stationary medium is $v$, the sphere will be opposed by a force calculated to be:

$$
F=P_{d} A
$$

If the area of the sphere is $4 \pi r^{2}$, the area that faces the liquid medium would be $2 \pi r^{2}$.

In hydrodynamics, the moving body opposed by dynamic pressure directly correlated with the medium density and with the square of the relative velocity, according to Action-Reaction of Newton Law which states if a force is applied to an object, there is an equal and opposite reaction. The opposite reaction measured to be:

$$
P_{d}=\frac{1}{2} \rho v^{2}
$$

where $\rho$ denotes the density of the medium and $v$ is the relative velocity of the moving object. Substituting the dynamic pressure value in Equation (9) and area values of $2 \pi r^{2}$, the opposing force on the sphere is obtained as follows:

$$
F=P_{d} A=\frac{1}{2} \rho v^{2} 2 \pi r^{2}=\rho v^{2} \pi r^{2} .
$$

We then multiply and divide the above equation by $t$ (time) to obtain

$$
F=\rho \pi r^{2} v t v / t .
$$

Since the speed $v$ multiplied by time $t$ is equal to space $L$ (length) and the velocity divided by $t$ is equivalent to acceleration $a$. Therefore, the area $\pi r^{2}$ times space $L$ is equal to volume $Q$. then opposing force on the sphere is obtained as follows:

$$
F=\rho \pi r^{2} L a=\rho Q a .
$$

Note that density multiplied volume is equal to mass, and therefore, we obtain

$$
F=m a
$$

\section{The Nature of Deceleration Force}

Newton's first law states that every object in a state of uniform motion tends to remain in that state of motion unless an external force is applied to it (Law of Inertia).

Therefore, even moving object has acceleration.

If an external force is applied on a freely moving object in order to decelerate, or change its direction, an equal inertial force acts on the object in an opposite direction of the applied force. What is the nature of the accelerating force that 
continuo to push the deceleration body?

Considering the vacuum as a fluid, due to interference drag the object drags a part of the medium and moves with it. When the velocity of the object is constant, the relative velocity of the dragged medium with the body will be zero. However, the moving vacuum continues to exercise accelerating force and keep the body moving. The moving body in space is surrounded by an ultra-high vacuum that navigate with it. At a short distance, the body's adjacent vacuum moving at the same speed of the body has no friction with the surrounding static vacuum therefore continues to move.

If external force stops the moving body, the adjacent moving vacuum continues to move, colliding with body particles and gives to it its momentum, creating radiation pressure, continue to push until the adjacent vacuum stop to move. When the body decelerates, a positive force acts on it and continue to push it in the same direction of the moving body.

The decelerating force will subtract a part of the moving body in a way that the sum of forces in a particular direction is equal to the mass of the object times acceleration; $\Sigma F=$ ma.

The force exercised on the moving object will be $F=\rho v^{2} A=m a$.

Where $\mathrm{v}$ is the relative velocity between the flowing vacuum and the object.

When the decelerating force will be the same as the acceleration force the body will stop completely.

\section{Reduced Acceleration on Long Distances}

Considering the vacuum as a fluid, any motion in inertial frame is resisted by inertia due to resistance of the fluid medium.

The movement of a fluid in a fluid medium imply a drag force known as interference drag which is proportional to the density of the fluid times the square of the relative velocity between static fluid and the moving fluid and the drag coefficient as it is expressed in the drag force equation;

$$
F_{d}=\frac{1}{2} \rho v^{2} A C_{D},
$$

where $F_{d}$ is the drag force, which by definition is the force component in the direction of the flow velocity, $\rho$ is the mass density of the fluid, $v$ is the relative velocity relative to the vacuum, $A$ is the contact area, and $C_{D}$ is the drag coefficient related to the specific fluid. The drag coefficient depends on the Reynolds number that is related to the viscosity between the moving fluid and the static fluid. As the Reynolds number increases, inertial forces become stronger than viscous forces and a laminar boundary layer is generated.

In fluid dynamics, the same drag equation is used to calculate the force of drag experienced by a flow of fluid through a fully enclosing static fluid. Therefor in the case of flowing photons in the vacuum, there is some resistance due to viscosity and elasticity of the medium, equivalent to magnetic permeability times electric permittivity. 
Therefore, the dragged medium will interact with the surrounding static medium creating a shear viscosity that expresses its resistance against shearing flows, where the adjacent layers move parallel to each other at different speeds.

The drag force equation is transformed to the pressure equation by dividing both sides by area to obtain:

$$
F_{d} / A=P=\frac{1}{2} \rho v^{2} C_{D}
$$

where $\rho$ is the density of the vacuum.

Where: $P$ is the pressure gradient generated by drag.

In the case of gravity force, where the vacuum density is $9.89^{-27} \mathrm{~kg} / \mathrm{m}^{3}, v=c=$ $3 \times 10^{8} \mathrm{~m} / \mathrm{s}$ and the drag coefficient is between 0.1 and 0.2 .

Substituting for $\rho, v^{2}$ and $C_{D}(0.13349)$ in Equation (14) gives:

$$
P=6.67383255 \times 10^{-11} \mathrm{~kg} / \mathrm{m} \cdot \mathrm{s}^{2} \text { or } \mathrm{N} / \mathrm{m}^{2}
$$

which is the same value of constant $G$ which is an expression of the magnitude of gravitation force reduction due to the drag interaction effect.

Therefore, a moving body in the outer space drags a part of the vacuum which travel at the same speed of the spacecraft, the dragged vacuum interacts with the static vacuum, and reduces its momentum. Although the momentum reduction is negligible, in a very long distances navigation, this effect could be significant. Such effect could be one of the explanation as both Pioneer 10 and Pioneer 11 space craft's navigation that escape the solar system. Upon very close examination of navigational data, the spacecraft was found to be slowing slightly more than expected. The effect is an extremely small acceleration towards the Sun, of $(8.74 \pm 1.33) \times 10^{-10} \mathrm{~m} / \mathrm{s}^{2}$, which is equivalent to a reduction of the outbound velocity by $1 \mathrm{~km} / \mathrm{h}$ over a period of ten years [18] [19] [20].

The spacecraft acceleration in the outer space depends on the density of the medium in which it travels. Therefore, the change of acceleration of the aircraft could be used to predict the vacuum density in different zones of the trajectory.

\section{Conclusions}

The nature and essence of the inertia are explained by considering the particle of the matter immersed in a continuous vacuum which behaves as a fluid.

The interaction between the moving object and the vacuum takes place in the quantum realm which can be described with classical hydrodynamic laws. Considering the vacuum density and applying classical hydrodynamics, analytical formulations are applied to calculate the force acting on the matter leaded to Newton law of inertia.

In the quantum realm, the inertia is caused by the effect of quantum vacuum energy on matter through the effect of ultra-high frequency radiation on matter. Since such radiation would penetrate any matter and act all over its constituent particles, not just its surface.

The gravitation mechanism was discussed in a previous article where it was 
explained by the effect of the interaction between the flowing virtual photons against inertial mass while the interaction of a moving mass against the static quantum vacuum creates inertia or acceleration. The common point between gravitation force and inertia is the interaction between matter and the vacuum.

Analytical formulations derived from Planck energy equation and Einstein energy equation were applied to calculate the radiation pressure which leaded the same formula of Newton second law. The radiation pressure is described as longitudinal waves with Pointing vector created by the moving body against static vacuum which creates the acceleration. The interaction between the moving body drags a part of the vacuum till it moves with it in this case the inertia will disappear. When the body stops, the moving vacuum continues to flow acting on the matter causing the deceleration till the point where there is no movement relative to the body, in this point the deceleration will vanish. Confirmation of this theory by experimental data will open a new direction to develop antigravity and anti-inertia machines and allows to drive Inertia to prevent many catastrophes such as accidents on highways, catastrophes on the railway, air crashes. Moreover, it would be possible to prevent collision of an asteroid with Earth.

\section{Conflicts of Interest}

The author declares no conflicts of interest regarding the publication of this paper.

\section{References}

[1] Isaac, N., Motte, A. and Chittenden, N.W. (1846) Newton's Principia: The Mathematical Principles of Natural Philosophy.

[2] Einstein, A. (1997) The Foundation of the General Theory of Relativity. Engel, A., Trans., Princeton University Press, Princeton, 57.

[3] Veldman, M.J.G. (1986) The Higgs Boson. Scientific American.

[4] Haisch, B., Rueda, A. and Puthoff, H. (1994) Inertia as a Zero-Point-Field Lorentz Force. Physical Review A, 49, 678-694. https://doi.org/10.1103/PhysRevA.49.678

[5] Bush, J.W.M. (2015) The New Wave of Pilot-Wave Theory. Physics Today, 68, 47-53. https://doi.org/10.1063/PT.3.2882

[6] Bush, J.W.M. (2015) Pilot-Wave Hydrodynamics. Annual Review of Fluid Mechanics, 47, 269-292. https://doi.org/10.1146/annurev-fluid-010814-014506

[7] de la Peña, L., Cetto, A.M. and Valdés-Hernandes, A. (2014) The Zero-Point Field and the Emergence of the Quantum. International Journal of Modern Physics E, 23, Article ID: 1450049. https://doi.org/10.1142/S0218301314500499

[8] de la Peña, L., Cetto, A.M. and Valdés-Hernandes, A. (2014) Theo M Nieuwenhuizen. In: Pombo, C., Furtado, C., Khrennikov, A.Y., Pedrosa, I.A. and Špička, V., Eds., Quantum Foundations and Open Quantum Systems, World Scientific, Singapore, 399-461. https://doi.org/10.1142/9789814616737 0011

[9] Grössing, G. (2014) Emergence of Quantum Mechanics from a Sub-Quantum Statistical Mechanics. International Journal of Modern Physics B, 28, Article ID: 1450179. https://doi.org/10.1142/S0217979214501793 
[10] Grössing, G. (2014) Emergence of Quantum Mechanics from a Sub-Quantum Statistical Mechanics. In: Pombo, C., Furtado, C., Khrennikov, A.Y., Pedrosa, I.A. and Špička, V., Eds., Quantum Foundations and Open Quantum Systems, World Scientific, Singapore, 375-398. https://doi.org/10.1142/9789814616737 0010

[11] Cambier, J.-L. (2009) Inertial Mass from Stochastic Electrodynamics. In: Millis, M. and Davis, E., Eds., Frontiers of Propulsion Science, American Institute of Aeronautics and Astronautics, Reston, 423-454. https://doi.org/10.2514/5.9781563479953.0423.0454

[12] Rueda, A. and Haisch, B. (1998) Inertia as Reaction of the Vacuum to Accelerated Motion. Physics Letters A, 240, 115-126. https://doi.org/10.1016/S0375-9601(98)00153-4

[13] Rueda, A. and Haisch, B. (1998) Contribution to Inertial Mass by Reaction of the Vacuum to Accelerated Motion. Foundations of Physics, 28, 1057-1108. https://doi.org/10.1023/A:1018893903079

[14] Maurice Dirac, P.A. (1930) A Theory of Electrons and Protons. Proceedings of the Royal Society of London, Series A, 126, 360-365. https://doi.org/10.1098/rspa.1930.0013

[15] Rauscher, E.A. (1968) Electron Interactions and Quantum Plasma Physics. Journal of Plasma Physics, 2, 517-541. https://doi.org/10.1017/S0022377800004013

[16] Rauscher, E.A. (2004) Dynamic Plasma Excitation Modes of Propagation in the Ionosphere. Princeton Architectural Press, Hudson, 13, 295.

[17] Matsas, G. (2002) The Fulling-Davies-Unruh Effect is Mandatory: The Proton's Testimony. International Journal of Modern Physics D, 11, 1573-1577. https://doi.org/10.1142/S0218271802002918

[18] Nieto, M.M. and Turyshev, S.G. (2004) Finding the Origin of the Pioneer Anomaly. Classical and Quantum Gravity, 21, 4005-4024. https://doi.org/10.1088/0264-9381/21/17/001

[19] Anderson, J.D., Laing, P.A., Lau, E.L., Liu, A.S., Nieto, M.M. and Turyshev, S.G. (1998) Indication, from Pioneer 10/11, Galileo, and Ulysses Data, of an Apparent Anomalous, Weak, Long-Range Acceleration. Physical Review Letters, 81, 2858-2861. https://doi.org/10.1103/PhysRevLett.81.2858

[20] Turyshev, S.G., Anderson, J.D., Laing, P.A., Lau, E.L., Liu, A.S. and Nieto, M.M. (1999) The Apparent Anomalous, Weak, Long-Range Acceleration of Pioneer 10 and 11. Proceedings of the 34th Rencontres de Moriond Meeting on Gravitational Waves and Experimental Gravity, Les Arcs, 23-30 January 1999, 481-486. arXiv: gr-qc/9903024. 